\title{
Fisioterapia no pós-operatório de correção artroscópica do impacto femoroacetabular
}

\author{
Physical therapy following femoroacetabular impingement arthroscopy \\ Viviane Bortoluzzi Frassona, Anete Beling Morales ${ }^{b}$, Anna Torresanc, Marcus Crestanid, \\ Paulo David Gusmão Fortes ${ }^{\mathrm{d}}$, Marco Aurélio Telöken ${ }^{\mathrm{d}}$, Marco Aurélio Vaz \\ a Fisioterapeuta. Physique - Centro de Fisioterapia, Porto Alegre, RS. \\ b Fisioterapeuta. Mestranda em Ciências do Movimento Humano, Universidade Federal do Rio Grande do Sul. Physique - Centro de Fisioterapia e \\ Atividade Física, Porto Alegre, RS. \\ c Fisioterapeuta. Hospital Moinhos de Vento - Serviço de Ortopedia e Traumatologia. Physique - Centro de Fisioterapia e Atividade Física, Porto Alegre, RS. \\ d Médico. Hospital Moinhos de Vento - Serviço de Ortopedia e Traumatologia, Porto Alegre, RS. \\ e Educador Físico. Laboratório de Pesquisa do Exercício, Universidade Federal do Rio Grande do Sul, Porto Alegre, RS.
}

RESUMO

Introdução: Diferentes métodos para ganho de amplitude de movimento do quadril operado, descarga de peso sobre o membro operado e de retorno ao esporte são atualmente baseados na experiência clínica de alguns grupos de pesquisadores do Impacto Femoroacetabular. Entretanto, o protocolo ideal de Fisioterapia no pós-operatório de correção artroscópica do Impacto Femoroacetabular ainda não está estabelecido na literatura.

Objetivo: Apresentar uma metodologia para a reabilitação pós-operatória do Impacto Femoroacetabular, descrevendo um protocolo com base na experiência dos autores e em conhecimentos biomecânicos.

Descrição da experiência: O protocolo de reabilitação proposto é dividido em quatro fases: (1) fase de reabilitação imediata, que compreende a fase hospitalar e as duas semanas iniciais da reabilitação; (2) fase de retorno às atividades de vida diária, que abrange o primeiro mês de pós-operatório; (3) fase de reforço muscular específico e treino proprioceptivo e; (4) fase de preparo final para o retorno ao esporte. Os autores procuram apresentar neste artigo as bases científicas para as condutas propostas em cada uma das fases.

Conclusão: O conhecimento da patologia, da biomecânica articular do quadril e das bases fisiológicas dos métodos e técnicas de fisioterapia é fundamental para propor um protocolo adequado na ausência de evidências científicas suficientes para dar suporte ao protocolo mais adequado.

Palavras-chave: fisioterapia; impacto femoroacetabular; artroscopia de quadril; reabilitação.

Introduction: Different methods for the gain in hip range of motion, loading of the operated limb and return to sports practice are based on the experience of a few research groups on femoroacetabular impingement. However, the ideal rehabilitation protocol for the femoroacetabular impingement arthroscopy has not been established in the literature.

Objective: To present a rehabilitation protocol based on the authors' experience of several years in the rehabilitation of the femoroacetabular impingement arthroscopy and also based on biomechanical knowledge.

Description of experience: The proposed rehabilitation protocol is divided in four phases: (1) immediate rehabilitation, comprising the hospital period and the first two weeks of rehabilitation; (2) phase of returning to the daily life activities, which encompasses the first month after surgery; (3) phase of muscle strengthening and proprioceptive training; and (4) final phase for the preparation of the patient for the return to sports practice. The authors try to present the scientific bases for the proposed rehabilitation program in each of the four proposed phases.

Conclusion: Knowledge of the pathology, of the hip joint biomechanics and of the physiological bases of the physical therapy techniques are fundamental in order to propose an adequate protocol in the absence of sufficient scientific evidences supporting the best or the most adequate protocol.

Keywords: physical therapy; femoroacetabular impingement; hip arthroscopy; rehabilitation. 


\section{INTRODUÇÃO}

O impacto femoroacetabular ou IFA é caracterizado pelo contato anormal entre a borda acetabular e a junção cérvico-cefálica do fêmur. O IFA pode ocorrer em amplitudes fisiológicas do quadril, em uma articulação com alteração morfológica, ou em amplitudes que excedam àquelas consideradas fisiológicas em uma articulação normal ${ }^{2,8}$. Dois tipos distintos de IFA tem sido descritos: o impacto tipo CAME (abreviatura originária da redução da palavra em inglês camshaft, que significa eixo de comando ou cilindro com formato irregular), que está relacionado com a retificação da região entre o colo e a cabeça femoral; e o impacto tipo Pincer, que resulta da alteração focal ou global da cobertura acetabular ${ }^{8,16,17}$. Existem evidências de que o IFA sintomático levará ao dano intra-articular e que sua progressão promoverá lesões do labrum e da cartilagem acetabular, tornando-o responsável pelo surgimento precoce da doença degenerativa do quadril ${ }^{14,20-22}$. Por isso, o IFA é considerado uma das principais causas de osteoartrite do quadril em pacientes jovens e ativos ${ }^{16,20-24}$.

Esta alteração morfológica promove reduções na mobilidade do quadril, na capacidade de produção de força muscular e na funcionalidade dos pacientes ${ }^{26-28}$. A principal alteração de mobilidade está associada à perda dos movimentos de rotação interna e flexão do quadril ${ }^{29}$. A capacidade de produção de força diminui na maior parte dos grupos musculares que atuam no quadril, exceto nos rotadores internos e nos extensores de quadril ${ }^{27}$. Essas reduções na força e na mobilidade parecem prejudicar as atividades de vida diária (AVDs) dos pacientes com IFA, já que alterações têm sido encontradas em atividades simples como sentar, agachar e caminhar ${ }^{30-31}$.

A persistência dos sintomas, a presença de lesões labrais ou condrais e limitações funcionais para atividades físicas ou do dia-a-dia podem ser indicações de tratamento cirúrgico ${ }^{2,23}$. Uma das técnicas cirúrgicas mais utilizadas atualmente é a artroscopia do quadril2,32,33. O objetivo principal da artroscopia é alívio dos sintomas dos pacientes e a melhora da amplitude de movimento do quadril através da remoção das áreas de conflito ósseo com a realização da osteocondroplastia do fêmur proximal e/ou rebordo acetabular, diminuindo o impacto entre estas estruturas ${ }^{5,8}$. Além disso, a preservação do lábrum é preconizada devido ao seu papel crucial na mecânica articular do quadril. O reparo do lábrum, quando necessário, é obtido através do uso de técnicas de sutura com âncoras e é frequentemente associado ao procedimento de remodelamento da estrutura óssea ${ }^{8}$.

O tratamento do IFA do quadril tem revelado grandes avanços com as técnicas por videoartroscopia, incluindo os programas de reabilitação no período pós-operatório (PO). O objetivo central da reabilitação é o retorno do paciente as funções normais e possibilidade de retorno a práticas esportivas ${ }^{13}$. No entanto, são poucas, na literatura, as evidências específicas sobre o melhor método de ganho de amplitude de movimento (ADM), força muscular e retorno ao esporte após a artroscopia do quadril ${ }^{6-8}$. O desafio da fisioterapia neste momento é definir um protocolo adequado que permita a reabilitação precoce sem prejudicar o processo normal de cicatrização dos tecidos.

Nos últimos anos, alguns trabalhos que apresentam programas de reabilitação de grupos especializados na reabilitação após a artroscopia do quadril têm sido publicados em periódicos internacionais ${ }^{6-15}$. Porém, existem divergências ou lacunas em cada um desses protocolos, de modo que as evidências existentes não nos permitem escolher um desses protocolos como sendo o ideal para o tratamento de pacientes com IFA. Além disso, até o presente momento nenhum relato de experiência sobre o tratamento do IFA embasado em prática clínica e em conhecimentos biomecânicos foi encontrado em publicações nacionais, dificultando o acesso de muitos fisioterapeutas que buscam informação a respeito do tema. Portanto, o presente artigo apresenta a experiência dos autores na reabilitação pósoperatória do IFA, descrevendo seu protocolo e procurando embasar as diferentes fases do mesmo na literatura específica existente na área, na prática clínica e em conhecimentos biomecânicos.

\section{DESCRIÇÃO DE EXPERIÊNCIA}

O programa de fisioterapia pós-operatória de artroscopia de quadril para tratamento do IFA proposto neste artigo foi elaborado baseado na experiência dos autores no atendimento de mais de 300 pacientes com diagnóstico clínico de IFA, submetidos à artroscopia por equipe médica especializada e acompanhados na Physique - Centro de Fisioterapia. A elaboração do protocolo iniciou em 2007 com a estruturação de um programa básico após discussão com a equipe de cirurgiões. O conhecimento científico sobre biomecânica da articulação do quadril, cicatrização dos tecidos, e as alterações mecânicas e morfológicas apresentadas nos pacientes com IFA foi fundmanteal para a estruturação inicial do protocolo. Nos anos seguintes, alguns grupos de pesquisadores dos Estados Unidos da América publicaram na literatura internacional artigos propondo protocolos específicos baseado na experiência dos seus Centros de Cirugia e Reabilitação do Quadril. O protocolo foi então adaptado segundo as evidências que foram apresentadas na literatura nos anos de 2007, 2010, 2011, 2012, 2013 e $2014^{6-15}$. 
O objetivo central do programa é o retorno do paciente as funções normais e possibilidade de retorno a práticas esportivas $^{13}$. A recuperação da ADM do quadril, o controle do edema e hematoma, a redução da inibição muscular, a obtenção do controle neuromuscular e proprioceptivo progressivo, o reforço muscular, o condicionamento cardiovascular e o preparo para o retorno ao esporte específico foram os pontos principais contemplados no programa de reabilitação no $\mathrm{PO}^{6,8,10,13}$.

O programa de fisioterapia pós-operatória foi dividido em quatro fases: (1) fase de reabilitação imediata, que compreende a fase hospitalar e as 2 semanas iniciais da reabilitação; (2) fase de retorno às AVDs, que abrange as primeiras 4 semanas de $\mathrm{PO}$; (3) fase de reforço muscular específico e treino proprioceptivo, que inicia concomitante ao final da fase 3 e persiste até $12^{\underline{a}}$ semana; (4) fase de preparo final para o retorno ao esporte, a partir da $12^{a}$ semana após a realização da cirurgia. A delimitação cronológica de cada fase serve didaticamente como um guia para a reabilitação, porém não é absoluta. A progressão entre as fases deve ser criteriosamente determinada e levar em conta a resposta de cada indivíduo.

Uma visão geral do programa de reabilitação empregado pelos autores encontra-se na Tabela 1. Os objetivos da fase 1 do programa de reabilitação incluem controlar o edema e o hematoma na região anterior do quadril (portais artroscópicos), promover a recuperação dos tecidos lesionados, recuperar as ADMs do quadril, combater a inibição muscular e iniciar o programa de estabilização lombopélvica.

A fase 2 compreende a recuperação da ADM do quadril operado e obtenção de controle neuromuscular do membro operado, possibilitando a realização das AVDs. Além disso, nessa fase se dá início ao trabalho proprioceptivo, à progressão da estabilização lombopélvica e o restabelecimento da marcha normal. O trabalho iniciado na fase 2 progride na fase 3, que tem por objetivo principal o reforço muscular específico, permitindo o retorno pleno do paciente às suas AVDs. O objetivo é centrado no reforço da musculatura do quadril e do membro inferior, no trabalho estabilizatório da região lombopélvica e no controle proprioceptivo.

Os objetivos da fase final do programa de reabilitação incluem progredir com o reforço muscular e trabalho de hipertrofia direcionado para a prática esportiva específica do paciente, o condicionamento cardiovascular e o controle da progressão de sobrecarga sobre quadril.

\section{DISCUSSÃO}

\section{Fase 1}

A fase 1 de reabilitação inicia-se no PO imediato, onde, ainda na sala de recuperação, o paciente inicia a utilização do aparelho de movimentação passiva contínua. Ainda na fase hospitalar o paciente inicia a movimentação ativa do membro operado e deve ser treinada a marcha com duas muletas. A fisioterapia na clínica deve iniciar imediatamente após a alta hospitalar, com frequência de três vezes na semana. Nestes dias o paciente deve ser treinado a executar exercícios que serão realizados também de forma domiciliar nos dias que o paciente não comparece à clínica.

O controle do edema e hematoma pode ser obtido através da utilização de crioterapia e laserterapia de baixa potência. O objetivo principal da crioterapia e da laserterapia é de diminuir a reação inflamatória inicial após o trauma cirúrgico ${ }^{35,36}$. Pesquisas realizadas na última década sugerem que a laserterapia de baixa potência produz efeitos anti-inflamatórios, contribuindo para o alívio da dor ${ }^{35}$. Os mecanismos de alívio da dor mediados pela laserterapia de baixa potência ainda não são totalmente compreendidos. Várias investigações explorando os efeitos da irradiação a laser nos tecidos pleiomórficos fornecem explicações plausíveis para os efeitos clínicos desta terapia. Efeitos anti-inflamatórios da irradiação do laser vermelho e infravermelho foram apresentados pela redução de marcadores inflamatórios específicos (prostaglandina E2, interleucina $1 \beta$, fator de necrose tumoral $\alpha$ ), em ensaios in vitro e estudos em animais in vivo ${ }^{37} \mathrm{e}$ no homem ${ }^{35,38}$.

A crioterapia tem sido amplamente usada após traumas ortopédicos e cirurgias há muitos anos ${ }^{39-42}$. Existem evidências na literatura de que a crioterapia diminui a dor e o edema ${ }^{42,43}$ por meio de muitos mecanismos, incluindo vasoconstrição ${ }^{43-45}$, redução da permeabilidade dos capilares e sangramento excessivo ${ }^{46}$; redução da exigência metabólica das células ${ }^{42,47}$, redução dos mediadores inflamatórios ${ }^{42,47}$ e redução da velocidade da condução nervosa motora e sensitiva $^{37,42}$. A crioterapia parece ter efeito benéfico no PO, diminuindo a temperatura intra-articular ${ }^{48}$.

A utilização da máquina de movimentação passiva contínua (MPC) já é bem estabelecida após cirurgias de joelho ${ }^{49,50}$. O objetivo principal do MPC é favorecer a nutrição da cartilagem (por meio do aumento da produção, circulação e reabsorção do líquido sinovial), diminuir o edema (ao aumentar o fluxo e retirada de líquidos articulares e periarticulares) e favorecer a recuperação das $\mathrm{ADMs}^{51}$. $\mathrm{Na}$ artroscopia do quadril o uso do MPC tem os mesmos objetivos $^{52}$. O movimento lento do quadril operado favorece a nutrição da cartilagem, facilita eliminação do edema articular e possibilita o ganho da amplitude de flexão do quadril de forma indolor ${ }^{50,52}$. O uso da máquina de MPC também é recomendada na tentativa de evitar aderências na articulação do quadril ${ }^{8}$. A Figura 1A demonstra a utilização do aparelho de movimentação passiva contínua. 
Tabela 1. Protocolo de fisioterapia utilizado na Physique para reabilitação após artroscopia do quadril.

\begin{tabular}{|c|c|c|c|c|c|c|c|c|c|c|}
\hline Semanas & 1 & 2 & 3 & 4 & 5 & 6-8 & 8-10 & $10-12$ & $12+$ & $16+$ \\
\hline \multicolumn{11}{|l|}{ Mobilidade } \\
\hline Aparelho de mobilização passiva contínua (MPC) & • & • & & & & & & & & \\
\hline Rotação interna e externa (com MO em flexão e extensão - DD e DV) & $\cdot$ & $\bullet$ & • & $\cdot$ & • & • & • & $\cdot$ & • & • \\
\hline \multicolumn{11}{|l|}{ Isométricos } \\
\hline Quadríceps/glúteos/isquiotibiais/adutores e abdutores & $\bullet$ & $\bullet$ & & & & & & & & \\
\hline \multicolumn{11}{|l|}{ Ativos } \\
\hline Flexores plantares e dorsais & • & & & & & & & & & \\
\hline Plantiflexão bilateral no plano em pé & • & & & & & & & & & \\
\hline Flexo-extensão de quadril e joelho & $\cdot$ & $\bullet$ & $\bullet$ & $\bullet$ & • & & & & & \\
\hline Flexão de quadril seguida de abdução do quadril & • & $\bullet$ & $\bullet$ & $\bullet$ & • & • & & & & \\
\hline Abdução do quadril com $\mathrm{MO}$ em extensão em DD & $\cdot$ & $\bullet$ & & & & & & & & \\
\hline Abdução de quadril em DL & & • & & & & & & & & \\
\hline Rotação externa em DL & & • & & & & & & & & \\
\hline Flexo-extensão de joelho sentado & • & & & & & & & & & \\
\hline Abdução do quadril em pé & • & & & & & & & & & \\
\hline Flexão de joelho em pé & • & & & & & & & & & \\
\hline \multicolumn{11}{|l|}{ Resistidos } \\
\hline Flexores plantares e dorsais & • & $\bullet$ & $\bullet$ & $\bullet$ & • & $\bullet$ & • & • & • & $\bullet$ \\
\hline Flexão-extensão de joelho com caneleira & & $\bullet$ & $\bullet$ & & & & & & & \\
\hline Abdução (DL, passada, slide) & & & • & • & • & $\bullet$ & • & • & $\bullet$ & • \\
\hline Abdução de quadril na máquina & & & & - & • & • & • & • & • & - \\
\hline Flexão de joelho na máquina & & & & - & • & • & • & • & • & - \\
\hline Extensão de joelho na máquina & & & & • & • & • & • & • & • & • \\
\hline Rotação externa em DL & & & • & • & • & • & • & • & - & - \\
\hline Agachamento (até $60^{\circ}$ de flexão de quadril) & & & & • & • & • & • & • & • & • \\
\hline Agachamento lateral & & & & & & • & • & • & $\cdot$ & $\bullet$ \\
\hline RE e RI em pé com quadril em EXT & & & & & & • & - & • & • & • \\
\hline \multicolumn{11}{|l|}{ Estabilização Central (CORE) } \\
\hline Isométrico de transverso abdômen/multífidos & • & • & & & & & & & & \\
\hline Prancha em DV & & & • & • & • & • & • & • & $\bullet$ & • \\
\hline Prancha em DL & & & & • & - & • & - & • & • & • \\
\hline Abdominais & & • & • & • & • & • & - & • & • & • \\
\hline Ponte & & • & • & • & & & & & & \\
\hline Ponte unipodal & & & & & • & - & - & - & - & - \\
\hline \multicolumn{11}{|l|}{ Exercícios Cardiovasculares } \\
\hline Bicicleta vertical & & & • & • & • & • & • & • & • & • \\
\hline Esteira ergométrica sem inclinação & & & & & • & • & • & • & • & • \\
\hline Aparelho elíptico & & & & & & & • & • & • & • \\
\hline Corrida & & & & & & & & • & $\bullet$ & • \\
\hline Natação & & & & • & • & • & - & • & • & • \\
\hline \multicolumn{11}{|l|}{ Propriocepção } \\
\hline Equilíbrio unipodal & & & • & & & & & & & \\
\hline Equilíbrio unipodal na cama elástica & & & • & - & • & • & • & & & \\
\hline Treino voltado para o esporte & & & & & & & & • & • & • \\
\hline Cama elástica, balancinho, meia bola, pranchas & & & • & - & • & • & • & • & & \\
\hline \multicolumn{11}{|l|}{ Flexibilidade } \\
\hline Isquiotibiais & $\cdot$ & $\bullet$ & $\bullet$ & • & $\bullet$ & • & • & • & • & • \\
\hline Quadríceps & • & $\bullet$ & $\bullet$ & - & $\bullet$ & - & • & • & $\bullet$ & • \\
\hline Flexores de quadril & & $\bullet$ & $\bullet$ & • & $\bullet$ & - & • & • & $\bullet$ & • \\
\hline Panturrilha & • & • & • & • & • & • & • & • & • & - \\
\hline \multicolumn{11}{|l|}{ Descarga de Peso/Marcha } \\
\hline Treino de marcha com muletas bilateralmente & • & & & & & & & & & \\
\hline Treino de marcha com uma muleta & & $\bullet$ & & & & & & & & \\
\hline Treino de marcha sem muletas & & & $\bullet$ & & & & & & & \\
\hline Descarga de peso ANT/POST e LAT/LAT & & & • & • & & & & & & \\
\hline
\end{tabular}

Obs.: Descarga de peso conforme tolerância durante $1{ }^{\underline{a}}$ semana.

$\mathrm{ANT}=$ anterior; $\mathrm{DD}=$ decúbito dorsal; $\mathrm{DV}=$ decúbito ventral; $\mathrm{DL}=$ decúbito lateral; $\mathrm{EXT}=$ extensão; FLX = flexão; LAT = lateral; $\mathrm{MO}=$ membro operado; POST = posterior; $\mathrm{RE}=$ rotação externa; $\mathrm{RI}=$ rotação interna 
O processo de recuperação das ADMs deve ser iniciado nesta fase com os exercícios ainda realizados no hospital. A melhora do movimento rotacional do quadril operado é um dos principais objetivos da fase inicial da reabilitação. A forma de trabalhar o ganho de ADM do quadril operado é assunto controverso nos protocolos propostos pela literatura, onde diferentes autores propõem restrições diversas ${ }^{6-15}$. O protocolo de ADM ideal deve basear-se no estresse produzido nas estruturas operadas (lábrum e cartilagem) que necessitam de um tempo para cicatrização sem a aplicação de grande magnitude de estresse. Em estudo realizado em cadáveres, Dy et al. ${ }^{53}$ demonstraram que torques abdutores e rotacionais aplicados em flexão e extensão do quadril causam estresse no lábrum, em especial na posição $2 \mathrm{~h}$. Para facilitar a localização do estresse radial no lábrum, é utilizada a analogia de um relógio, onde $12 \mathrm{~h}$ corresponde à superior e $3 \mathrm{~h}$ à anterior. A posição $2 \mathrm{~h}$ corresponde a região anterosuperior do lábrum, local onde acontece a maioria das lesões causadas pelo impacto tipo $\mathrm{CAME}^{54}$. Maiores cargas são impostas na região anterior do labrum durante o movimento de flexão e adução do quadril, principalmente quando o quadril estiver posicionado em $90^{\circ}$ de flexão. A rotação externa, por sua vez, quando realizada na posição neutra de flexão-extensão do quadril, como por exemplo em decúbito ventral, reduz as cargas no labrum antero-lateral ${ }^{53,54}$. Dados precisos da angulação e do estresse produzido nas estruturas do quadril com diferentes ADMs ainda são insuficientes. Por isso, cuidados adicionais são necessários em pacientes com sutura labral.

Diversas propostas de recuperação de ADM são encontradas na literatura (Tabela 2). Apesar da heterogeneidade das informações quanto aos cuidados da mobilização articular observados na Tabela 2, podemos basear a progressão da mobilidade nos princípios biomecânicos.
Logicamente, movimentos que provoquem o estresse na região onde foi realizada a sutura labral devem ser evitados para aumentar as chances de uma cicatrização satisfatória ${ }^{54}$. Preconizamos que o ganho de ADM do quadril seja feito de forma progressiva, baseado na tolerância do paciente e tendo como objetivo final a recuperação completa das ADMs do quadril operado até o final do primeiro mês de $\mathrm{PO}$. Alguns exercícios utilizados para ganho de ADM podem ser visualizados na Figura 1A, 1B e 2D.

O combate à inibição muscular deve iniciar imediatamente através de medidas simples como o combate ao edema e à dor. Geralmente a musculatura glútea, que já está fraca ou inibida no pré-operatório, perde ainda mais força devido ao desuso e ao edema provocados pelo procedimento cirúrgico ${ }^{5,56}$. Alguns autores já demonstraram que o grau de inibição muscular é proporcional ao aumento de volume na articulação $0^{57}$. Especificamente na articulação do quadril, Freeman et al. ${ }^{56}$ verificaram que ocorreu a redução da atividade eletromiográfica do glúteo máximo após a introdução de uma solução salina intra-articular. Portanto, o combate ao edema com medidas anti-inflamatórias é fator primordial para se evitar a inibição muscular. Associado a isto, exercícios que utilizem a musculatura do membro operado através de contrações isométricas ou exercícios ativos livres iniciais com pequenas alavancas são fundamentais no despertar muscular.

Sabe-se que os abdutores do quadril tem papel fundamental na marcha ${ }^{30}$. A inibição da musculatura abdutora leva ao estresse na articulação operada durante o apoio unipodal da marcha. Por esse motivo, os músculos abdutores do quadril devem ser a preocupação inicial do fisioterapeuta no processo de reabilitação. O glúteo médio é um músculo importante na reabilitação do indivíduo com IFA. Se estiver fraca ou for facilmente fatigável, esta musculatura pode ser responsável por uma inclinação pélvica excessiva e

Tabela 2. Restrições de amplitude de movimento pós artroscopia do quadril encontradas na literatura.

\begin{tabular}{|c|c|c|c|}
\hline Procedimento & Osteoplastia & Condroplastia & Lesão Labral \\
\hline Wahoff e Ryan & Nenhuma & Nenhuma & $\begin{array}{l}\text { FLX } 120^{\circ}, \mathrm{ABD} 45^{\circ} \text {, sem RE por } 17-21 \text { dias, sem EXT } \\
\text { por } 17-21 \text { dias e EXT } 0^{\circ} \text { por mais } 1 \text { semana }\end{array}$ \\
\hline Spencer-Gardner et al. & Nenhuma & Não cita & $\begin{array}{l}\text { Hiperextensão e RE limitadas em } 20^{\circ} \text { de } \\
3 \text { a } 4 \text { semanas }\end{array}$ \\
\hline Stalzer et al. & $90^{\circ}$ de flexão por 10 dias & $90^{\circ}$ de flexão por 10 dias & $\begin{array}{l}90^{\circ} \text { de flexão por } 10 \text { dias, } 25^{\circ} \text { de ABD por } 3 \text { semanas, } \\
\text { RE e EXT leve por } 3 \text { semanas; RI livre }\end{array}$ \\
\hline Enseki et al. & $\begin{array}{l}\text { Evitar ADM excessiva de FLX e } \\
\text { ABD por } 2 \text { semanas }\end{array}$ & Não cita & Evitar ADM excessiva de FLX e ABD por 2 semanas \\
\hline Bennel et al. & $\begin{array}{l}\text { Evitar ADM excessiva de FLX, } \\
\text { ABD e rotações }\end{array}$ & $\begin{array}{l}\text { Se microfratura, RE somente } \\
\text { entre } 4 \text { e } 7 \text { semanas }\end{array}$ & $\begin{array}{l}\text { Evitar FLX além de } 90^{\circ} \text { por } 6 \text { semanas; } \\
\text { ADM excessiva de ABD e rotações }\end{array}$ \\
\hline Edelstein et al. & Nenhuma & Nenhuma & $\begin{array}{l}\text { Conforme a tolerância; se reparo capsular RE limitada } \\
\text { em } 30^{\circ} \text { e extensão em } 0^{\circ} \text { por } 6 \text { semanas. }\end{array}$ \\
\hline Cheatham e Kolber & Nenhuma & Não cita & Não cita \\
\hline
\end{tabular}

$\mathrm{ABB}=$ abdução; $\mathrm{ADM}=$ amplitude de movimento; $\mathrm{FLX}=$ flexão; $\mathrm{RE}=$ rotação externa; $\mathrm{RI}=$ rotação interna 

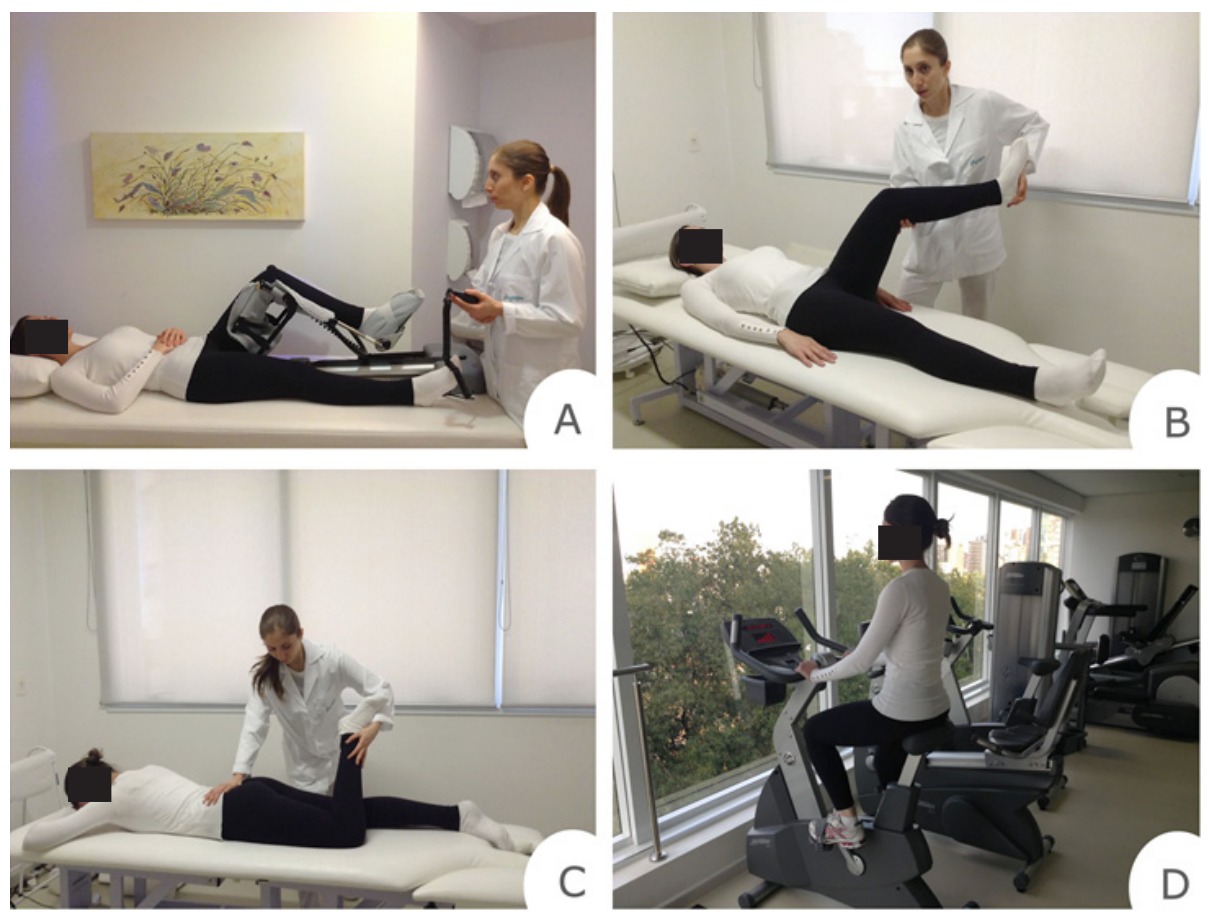

Figura 1. A) Uso do aparelho de mobilização passiva contínua (MPC); B) Mobilização passiva para ganho de rotação interna do quadril; C) Mobilização passiva para ganho de rotação externa do quadril; D) Uso da bicicleta estacionária vertical.
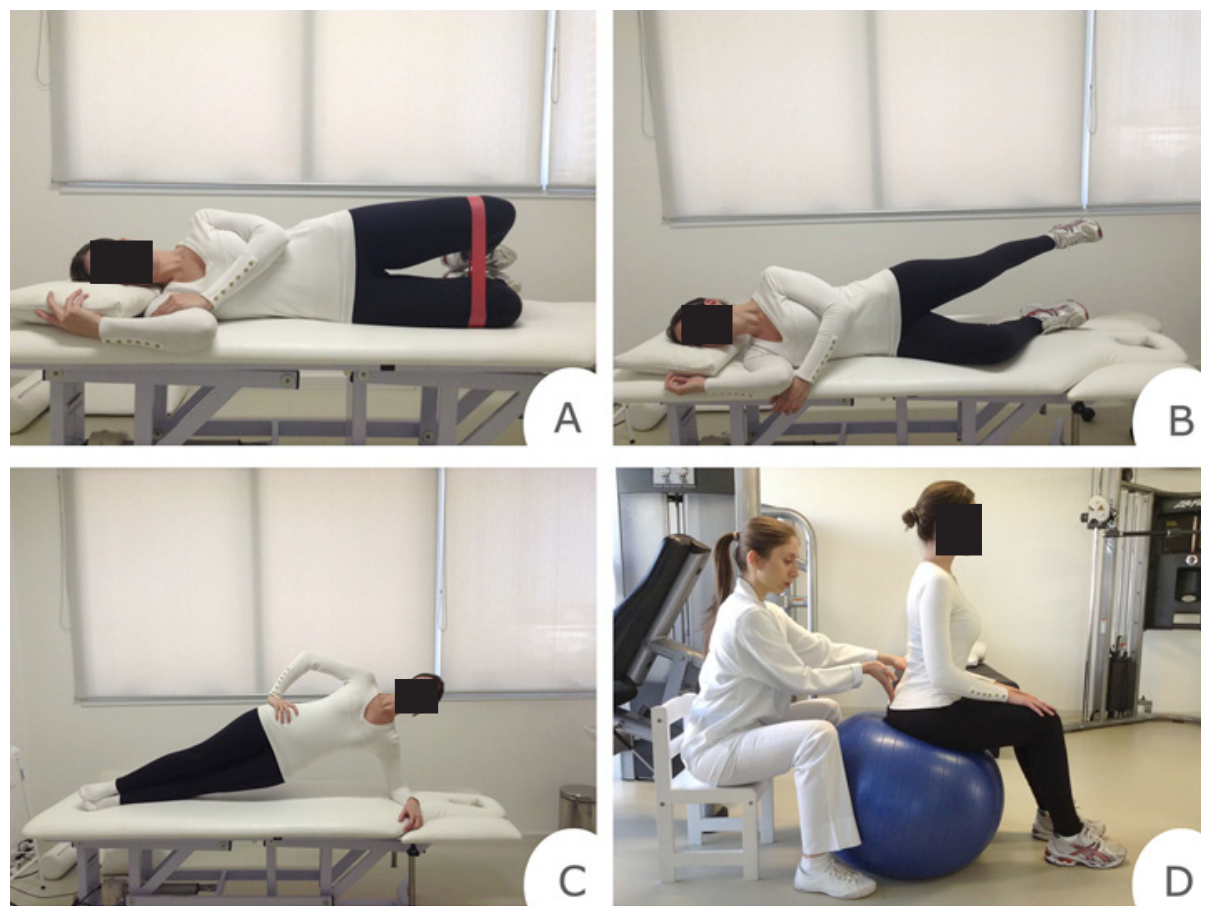

Figura 2. A) Rotação externa do quadril com faixa elástica e posição neutra; B) Abdução do quadril em decúbito lateral; C) Prancha lateral; D) Mobilidade pélvica com auxílio de fisioterapeuta sobre bola.

rotação femoral interna, provocando um desalinhamento de todo o membro inferior ${ }^{55}$. Alguns estudos já avaliaram os melhores exercícios para ativar os músculos abdutores do quadril através da eletromiografia ${ }^{55,58}$. Dentre os exercícios selecionados estão o de abdução em decúbito lateral, com o quadril em posição neutra (Figura 2B), o deslocamento lateral 
com faixa elástica (Figura 3D) e a ponte em apoio unipodal ${ }^{58}$. Respeitando a tolerância do paciente, estes exercícios são fundamentais e devem ser inseridos ao longo do processo de reabilitação. Outro grupo muscular crucial na reabilitação do paciente com IFA é o dos rotadores externos do quadril, chamados pelvitrocanterianos. Esses músculos possuem um braço de alavanca curto e, portanto, têm o potencial de agir como estabilizadores profundos da articulação coxofemoral. Por isso, devem ser retreinados e fortalecidos desde o início da reabilitação ${ }^{13}$. Um dos exemplos de exercício utilizado para fortalecer esta musculatura é a rotação externa do quadril em decúbito lateral com o uso de faixa elástica (Figura 2A).

Nesta fase, também se iniciam os exercícios de estabilização lombopélvica através do aprendizado inicial da contração abdominal (em especial do músculo transverso do abdômen). O programa de estabilização utilizado segue as diretrizes propostas por Richardson et al. ${ }^{59}$. Os exercícios de estabilização central têm por objetivos melhorar o controle postural dinâmico, garantir controle muscular apropriado do complexo lombopélvico, promover estabilidade proximal para movimentos eficientes das extremidades, além de estabelecer um programa de reabilitação abrangente e funcional ${ }^{60,61}$

A limitação articular provocada pelo IFA leva à alteração dos comprimentos das musculaturas periarticulares e modifica a dinâmica do quadril ${ }^{17}$. Os exercícios de flexibilidade têm a finalidade de garantir a manutenção do comprimento muscular e evitar contraturas articulares, e devem ser iniciados de forma suave na fase inicial da reabilitação. $\mathrm{O}$ alongamento dos flexores plantares e da musculatura posterior de coxa (isquiotibiais) deve ser realizado evitando o encurtamento que pode trazer prejuízos ao funcionamento mecânico adequado do quadril. Além disso, o alongamento quadriciptal e da musculatura flexora do quadril deve iniciar lentamente nesta fase, evitando assim o desenvolvimento de contraturas em flexão do quadril que prejudicam o funcionamento articular em fases de maior demanda ao longo da reabilitação.

Dor na região anterior da coxa ou tendinopatia do iliopsoas podem acontecer no PO de artroscopia do quadril ${ }^{55}$. Uma orientação interessante que pode ser dada aos pacientes é deitar-se em decúbito ventral por alguns períodos durante o dia, promovendo com isso o alongamento das estruturas anteriores do quadril operado.

Alguns cuidados são fundamentais na fase inicial da reabilitação como: (1) evitar forçar ADMs em amplitudes dolorosas devido ao risco de sobrecarregar as estruturas labrais e osteoarticulares que estão em processo de cicatrização durante o primeiro mês de $\mathrm{PO}$; (2) evitar excesso de atividades de impacto como caminhada, pois o quadril nesta fase inicial está sensível à carga devido ao processo inflamatório inerente ao trauma cirúrgico; (3) evitar posições mantidas por longos períodos que levam à rigidez dos tecidos, como a posição sentada. A sustentação do membro operado em flexão, a fim de mantê-lo suspenso, não é indicado pois pode acarretar na irritação do tendão do músculo iliopsoas que, como flexor do quadril, atua na manutenção desta posição ${ }^{8}$.

O treino de marcha durante esta fase inicial deve ser associado ao uso de muletas. Diferentes propostas de descarga de peso são encontradas na literatura no PO de artroscopia do quadril6-15. Alguns autores têm proposto protocolos de apoio parcial por 2 semanas seguido de apoio total conforme tolerância mesmo nos casos de reparo do lábrum ${ }^{62}$. Na maioria dos casos, o período no qual as muletas devem ser utilizadas é imprecisamente descrito na literatura. O apoio do membro inferior operado conforme a tolerância não interfere negativamente na recuperação após a artroscopia de quadril, e, por isso, o uso de muletas pode ser descontinuado de acordo com a condição do paciente ${ }^{63}$. No caso dos procedimentos em que foram realizados o reparo capsular ou microfraturas deve-se combinar com o cirurgião quando será iniciada a descarga de peso do membro operado, já que estas condições podem restringir o apoio precoce. De qualquer forma, o paciente deve ser encorajado à simular a passada normal com o uso das muletas mesmo se não for possível a descarga do peso na perna operada. Temos rotineiramente utilizado o apoio imediato conforme tolerância nos casos de osteocondroplastia com e sem sutura labral (as muletas são mantidas por no máximo 2 semanas).

\section{Fase 2}

A fase 2 de reabilitação compreende as duas semanas seguintes à fase inicial, sendo que, ao final desta fase, o paciente completará um mês de PO. Nesta fase o foco principal é tornar o paciente apto a realizar todas as AVDs que realizava antes da cirurgia como: caminhar normalmente, subir e descer escadas, dirigir e trabalhar. Este trabalho é feito na clínica de fisioterapia com a mesma frequência da fase 1 , ou seja, 3 vezes na semana. Nesta fase já deve ter ocorrido a redução do processo inflamatório inicial, e a absorção do edema e do hematoma. Caso ainda exista hematoma persistente no local, o uso de calor superficial é indicado para aumentar a circulação local e favorecer a sua reabsorçãa ${ }^{64}$.

O processo de recuperação da ADM deve ser continuado nesta fase, para que, ao final do 10 mês de $\mathrm{PO}$, o paciente tenha adquirido ADM superior ao pré-operatório de forma indolor. O aumento de 5 a 15 graus de rotações, segundo estudos atuais ${ }^{65,66}$, pode ser obtido após o procedimento cirúrgico. Exercícios assistidos ou passivos levando a 
articulação até o limite indolor em diferentes posições devem ser realizados com objetivo de atingir a melhor ADM possível de acordo com os valores prévios ao período PO. Os exercícios de flexibilidade continuam nesta fase com a mesma musculatura trabalhada na fase anterior, com ênfase no grupo muscular flexor do quadril.

O movimento cíclico articular, que na fase anterior era obtido através do aparelho de MPC, é agora realizado com a bicicleta ergométrica sem carga e banco em posição elevada ${ }^{14}$. A bicicleta utilizada deve ser a vertical, evitando assim a hiperflexão do quadril operado que pode ser motivo de dor e desconforto na região anterior do quadril $6,9,10$. A utilização da bicicleta vertical, com banco alto e sem carga, é estimulada precocemente nas primeiras semanas pela maioria dos protocolos ${ }^{6-15}$. A regulagem do assento na posição alta evita que o quadril seja mantindo em flexão excessiva (Figura 1D). O objetivo da bicicleta é promover o movimento cíclico articular que parece favorecer a nutrição cartilaginosa através de sua compressão e descompressão, auxiliando também na recuperação da ADM ativa de maneira indolor. Além disso, o trabalho ativo gerado pela musculatura dos membros inferiores favorece o controle muscular e o retorno da movimentação ativa normal dos membros inferiores.

Os exercícios ativos livres passam a ser enfatizados nesta fase com o objetivo de ganho de controle neuromuscular e preparo para o reforço muscular que iniciará na fase seguinte. O trabalho ativo de abdução, extensão e rotação do quadril deve ser realizado neste período. O reforço do membro inferior, que não foi diretamente afetado pela cirurgia e que não determina carga excessiva na articulação do quadril, pode ser realizado através de exercícios utilizando caneleiras e faixas elásticas de forma associada.

O trabalho estabilizatório lombopélvico deve progredir neste período com exercícios de controle da musculatura profunda da região. A contração do músculo transverso do abdômen, que foi treinada inicialmente em posições estáveis, passa a ser treinada em posições de maior demanda muscular e instabilidade. Sempre associado ao treino estabilizatório é exigido do paciente um bom posicionamento da região lombar e pélvica (pelve neutra e manutenção da curvatura lombar). Este trabalho é fundamental não somente no sentido de recuperação pós-operatória, mas também pelo fato da relação existente entre dor lombar e limitação da rotação do quadril, presente nos pacientes com IFA ${ }^{67,68}$. A reeducação neuromuscular dos estabilizadores da pelve e da coluna lombar é uma das bases da reabilitação pós artroscopia do quadril, principalmente em indivíduos com pretensões esportivas. A habilidade de manter a coluna lombar e a pelve em posição neutra é incentivada inicialmente em posições sem descarga de peso nos membros, como, por exemplo, com o indivíduo em decúbito, progridindo gradualmente para a posição sentada, em pé e durante atividades funcionais e esportivas ${ }^{17}$.

Na nossa experiência, encontramos dor lombar em pacientes com IFA que apresentam limitação da rotação do quadril. Alguns estudos já têm abordado este assunto e estabelecido uma relação entre dor lombar e limitação das rotações do quadril ${ }^{67-69}$. Van Dillen et al..$^{70}$ avaliaram a ocorrência de dor lombar em indivíduos participantes de atividades físicas com alta demanda rotacional, como o tênis e o golfe, e verificaram que o grupo com dor lombar apresentava assimetria rotacional e menos rotação total do quadril. Os movimentos repetidos assimétricos seriam o fator desencadeante de dor lombar. Almeida et al. ${ }^{71}$ investigaram dor lombar em atletas de judô e encontraram que, no grupo com dor, havia diminuição da rotação interna de quadril passiva e ativa no lado não-dominante e redução da rotação interna passiva do lado dominante, também demonstrando assimetria rotacional entre os membros inferiores. Além disso, segundo Watanabe et al. ${ }^{69}$, uma retificação da coluna lombar pode acontecer pela maior tendência à retroversão pélvica adotada por esses pacientes, gerando uma consequente diminuição da cobertura acetabular. Isto pode ocorrer a fim de minimizar o IFA dos pacientes, uma vez que promove menor contato contra o acetábulo. Entretanto, a retificação da coluna lombar à longo prazo pode ser responsável por provocar dor nessa região.

Nesta fase se inicia o treino de marcha sem muletas, quando o paciente é incentivado a deambular sem auxílio e a obter controle proprioceptivo suficiente para o apoio unipodal estável. O treino de equilíbrio em solo estável, progredindo de bipodal para unipodal, é realizado. O paciente é incentivado a iniciar caminhadas curtas no dia-a-dia. O paciente também deve ser treinado para evitar vícios de marcha com claudicação, diminuindo o tempo de apoio na perna operada, que é muito comum. Exercícios de transferência de peso entre os membros inferiores e que enfatizem as fases da marcha de forma sequencial são empregados para esta finalidade.

Ao final deste mês o paciente estará apto a retornar às AVDs que exigem caminhadas, sentar e levantar de cadeiras, escadas e trocas de postura. Cuidados devem ser tomados para evitar excessos neste período. A progressão da reabilitação determina uma sensação de bem-estar e de capacidade de realizar atividades sem dor. Esta sensação de bem-estar pode levar o paciente a cometer excessos como caminhadas longas, períodos longos na posição sentada e excessos na utilização de escadas. Estes excessos podem determinar o surgimento de dores na região do quadril 
operado muitas vezes de origem miofasciais devido à fadiga e uso inadequado da musculatura ainda enfraquecida do quadril.

Outro ponto importante a ser considerado nesta fase é que a fraqueza da musculatura glútea ainda presente, pode predispor ao desequilíbrio em relação à musculatura flexora do quadril levando à tendinopatia ${ }^{55}$. Para evitar agravar ou desenvolver a tendinite do músculo iliopsoas, exercícios com sobrecarga exagerada dos flexores do quadril devem ser evitados nas fases iniciais do $\mathrm{PO}^{55}$. O exercício de elevação da perna com o joelho estendido, por exemplo, mesmo que sem carga, não é realizado.

Caso o paciente apresente dores miofasciais neste período, técnicas de pressão inibitória de pontos dolorosos ${ }^{72}$, terapia combinada ${ }^{73}$, massoterapia ${ }^{13}$, e calor ${ }^{64}$ são boas opções terapêuticas e podem ser utilizadas. Os principais músculos que se apresentam hipersensíveis nesta fase são: tensor da fáscia lata e trato iliotibial, glúteos, pectíneo e iliopsoas ${ }^{6}$. A mobilização dos tecidos moles através das técnicas de terapia manual é endereçada ao alívio da dor oriunda de pontos gatilhos miofasciais. Se não tratados, os pontos gatilhos miofasciais podem ser resposáveis por dor crônica persistente ${ }^{72}$. Um estudo randomizado controlado realizado por Renan-Ordine et al. ${ }^{74}$, forneceu evidências de que a utilização conjunta de técnicas de terapia manual auxiliam no controle da dor musculoesquelética.

Os critérios para evolução para a fase seguinte são: ADM de $120^{\circ}$ de flexão e $45^{\circ}$ de rotação de quadril, capacidade de realizar exercícios ativos livres do quadril com grande alavanca (determina o despertar muscular), diminuição do edema e hematoma, da dor e desconforto mínimo durante e após a realização dos exercícios.

\section{Fase 3}

A fase do reforço muscular específico inicia-se com o paciente em torno do 10 mês de PO, quando o paciente está retornando plenamente às suas AVDs, podendo estenderse até a $12^{a}$ semana. O objetivo agora estará centrado no reforço da musculatura do quadril e do membro inferior, no trabalho estabilizatório da região lombopélvica e no controle proprioceptivo.

O trabalho em bicicleta ergométrica progride e o paciente pode iniciar a utilização de carga durante a utilização da mesma de forma progressiva. O objetivo deste trabalho é o aumento da resistência muscular localizada dos membros inferiores e o condicionamento cardiovascular do paciente em uma atividade com impacto reduzido no quadril. Neste momento podem ter início atividades como caminhada em esteira de maneira gradual e progressiva. A proposta é a alternância entre a bicicleta e a esteira, evitando a posição sentada por longos períodos, e o impacto acentuado da marcha. A bicicleta ergométrica deve ser mantida devido a sua função cíclica sobre a nutrição cartilaginosa, e a esteira prepara o quadril para receber impactos maiores com o passar do tempo. Após completar 1 mês de PO, o paciente pode ser liberado para recomeçar a nadar, se assim o desejar, desde que sejam realizadas somente as modalidades crawl e costas. A partir do 2o mês de cirurgia, o trabalho aeróbico poderá incluir a utilização do aparelho elíptico.

O reforço muscular específico progride com a utilização de faixas elásticas, caneleiras ou aparelhos de musculação. Nesta fase se utiliza trabalho inicial focado em resistência muscular localizada (séries de 12 a 15 repetições com carga de $60 \%$ de uma repetição máxima). O trabalho de hipertrofia muscular progredirá ao longo do próximo mês de PO, quando se aumentará a carga utilizada $(80 \%$ de 1 repetição máxima) e se diminuirá o número de repetições para 8 a $12^{75}$. Podem ser utilizados aparelhos como as máquinas flexoras e extensoras do joelho, e máquinas adutoras e abdutoras com carga progressiva. Cuidado especial deve ser observado com as máquinas adutoras e abdutoras para trabalhar em amplitude limitada, evitando posições extremas de quadril. Considera-se importante o trabalho de força dos seguintes grupos musculares: flexores e extensores do joelho, flexores plantares e dorsais do tornozelo, abdutores e adutores do quadril, extensores do quadril, rotadores externos e internos do quadril. A força destes grupos musculares proporciona o bom funcionamento mecânico do membro inferior e minimiza a sobrecarga articular excessiva durante atividades de impacto como a marcha. O trabalho de flexores do quadril deve vir associado à estabilização pélvica adequada, evitando o desenvolvimento de dor anterior no quadril. Exercícios avançados de estabilização lombopélvica são utilizados neste período como pranchas frontais e laterais ilustrados na Figura 2C.

O trabalho proprioceptivo progride quando os pacientes iniciam o treinamento em planos instáveis como cama elástica, balanços e pranchas proprioceptivas (Figura 3C). O objetivo principal do treino é que o paciente esteja apto a suportar a carga do apoio unipodal associado à desequilíbrios, trabalhando então a capacidade de reação dos membros. Trilhas proprioceptivas e recursos externos como bolas, bastões e faixas são utilizados para gerar desequilíbrios de forma progressiva. O foco principal nesta fase é no bom alinhamento do membro inferior e da coluna durante os exercícios, bem como com a capacidade de manter as posições em resposta aos desequilíbrios provocados. O trabalho rotacional sobre o quadril afetado é importante neste período. O paciente tem que estar apto ao controle rotacional sobre descarga de peso em planos instáveis. 
O treino de flexibilidade é mantido nesta fase com o objetivo de manutenção das ADMs do quadril obtidas nas fases anteriores. Neste sentido, são realizados exercícios passivos de rotação, flexo-extensão e abdução do quadril operado. Também são realizados alongamentos gerais dos flexores plantares do tornozelo, extensores e flexores de joelho e da musculatura anterior do quadril.

Os critérios para evolução para a fase seguinte são: força equivalente a $80 \%$ do lado contralateral (saudável), ADM completa do quadril operado, quadril indolor durante e após a fisioterapia e controle unipodal apropriado em plano instável.

\section{Fase 4}

Muitos pacientes que não apresentam motivação esportiva não realizam a fase 4 , correspondente ao final de tratamento. O preparo ao retorno ao esporte inicia em torno da $12^{\text {a }}$ semana de reabilitação, momento no qual o indivíduo é liberado para recomeçar a correr.

Os exercícios de reforço muscular localizado continuam sendo realizados na sala de musculação com auxílio de aparelhos. O treino estabilizatório do tronco também é mantido, progredindo até exercícios de elevado grau de dificuldade que combinam força, controle e coordenação do tronco neste período. Exercícios que envolvem corridas, mudanças de direção, saltos e giros são utilizados para preparação para o esporte (Figura 3A e 3B).

Muitos pacientes almejam o retorno à prática de corrida. A avaliação da possibilidade do retorno à corrida deve ser realizada pelo cirurgião de acordo com o estado da articulação encontrado no ato cirúrgico. Pacientes com desgastes no quadril são desencorajados a continuar com a corrida. O nosso protocolo de retorno à corrida consiste em iniciar progressivamente na esteira, sem inclinação, com a alternância entre alguns minutos de caminhada e corrida lenta. Num primeiro momento, o tempo de caminhada será três vezes maior que o tempo de corrida (três minutos de caminhada para um minuto de corrida). O tempo total no qual este revezamento será aplicado também deverá ser gradativamente ampliado até ser alcançado o tempo ideal para o paciente. É importante que o indivíduo não sinta dor durante a realização da atividade.

O retorno gradual às atividades esportivas é fator de sucesso nesta fase do tratamento. O retorno rápido às atividades de impacto como caminhada, corrida e atividades com saltos e giros sobre o membro operado levam ao surgimento de dores articulares e miofasciais que impossibilitam o retorno ao esporte. Geralmente, a liberação para esportes de contato, como futebol, vôlei, basquete e artes marciais ocorerrá a partir do 5으 mês de PO.
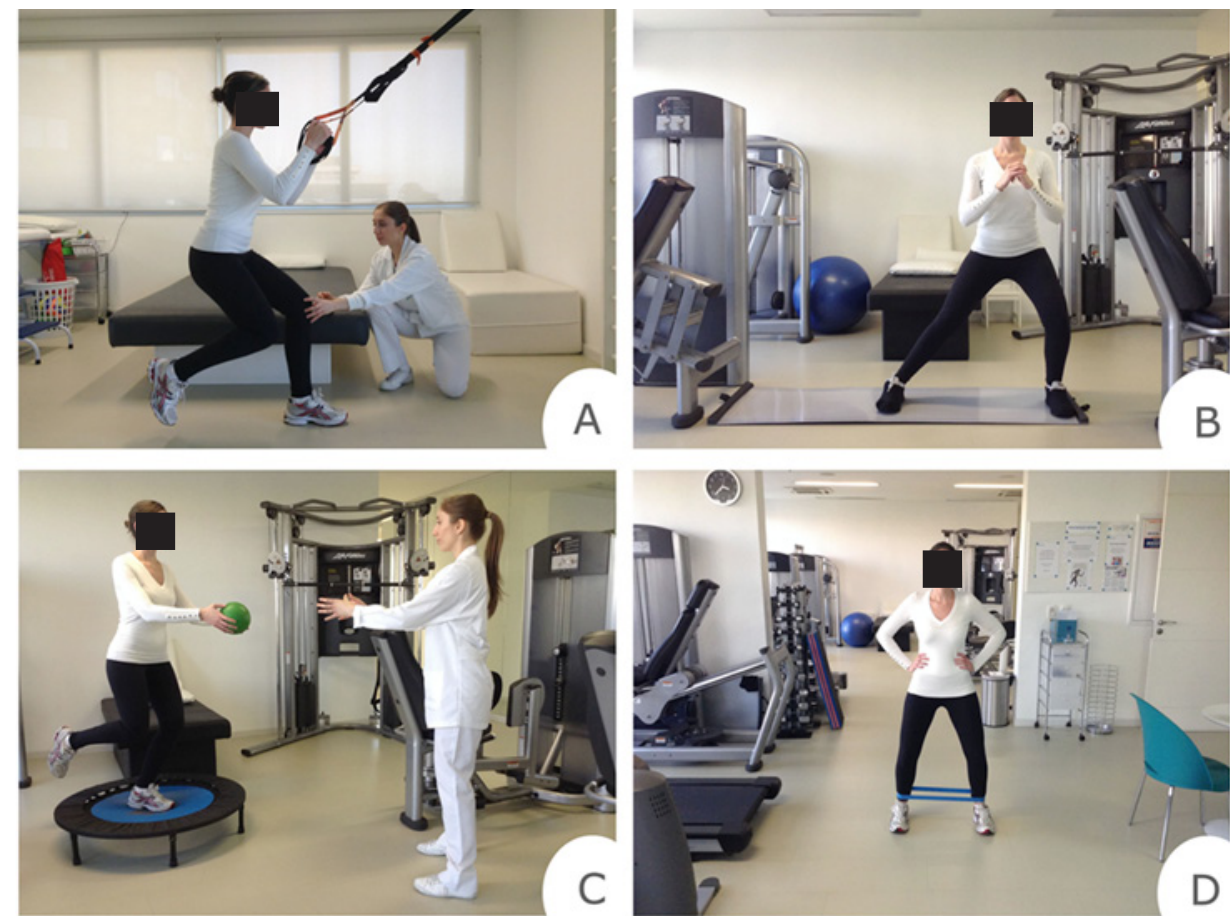

Figura 3. A) Agachamento unipodal com o uso de fita de suspensão; B) Exercícios em prancha deslizante; C) Propriocepção na cama elástica em apoio unipodal jogando bola; D) Deslocamento lateral com uso de faixa elástica. 
Os critérios utilizados para o retorno ao esporte são: quadril indolor, força equivalente a $90 \%$ a do lado oposto saudável, condicionamento cardiovascular igual ao nível pré-lesão, bons resultados em testes de agilidade e controle neuromuscular.

\section{CONCLUSÃO}

O número de cirurgias artroscópicas do quadril vem aumentando nos últimos anos devido a uma maior compreensão da patomecânica do IFA, das lesões labrais, e da sua relação com coxoartroses precoces ${ }^{1,2}$. Este fato está associado à evolução do exame clínico e complementar, favorecendo a realização do diagnóstico precoce de IFA ${ }^{3,4}$. A literatura tem revelado resultados animadores com este tipo de tratamento, principalmente quanto à melhora dos sintomas ${ }^{5}$.

Apesar da literatura crescente a respeito do IFA, a melhor evidência atual para a reabilitação pós-operatória da artroscopia do quadril de pacientes com IFA persiste baseada na experiência do profissional. Os protocolos descritos na literatura ainda divergem quanto ao apoio do membro operado e à progressão das ADMs. A maioria das técnicas fisioterapêuticas empregadas é baseada somente na cronologia da cicatrização dos tecidos abordados no procedimento. Como ainda não existem evidências suficientes de qual protocolo é superior, nosso serviço criou um programa de reabilitação pós artroscopia de quadril baseado nos conhecimentos biomecânicos e cinesiológicos e na sua vasta experiência clínica. A publicação deste relato de experiência e do nosso protocolo servirá para auxiliar outros fisioterapeutas em sua prática clínica e estimular a discussão sobre qual a forma mais adequada de conduzir a reabilitação desta condição tão específica.

\section{REFERÊNCIAS}

1. Martin RL, Enseki KR, Draovitch P, Trapuzzano T, Philippon MJ. Acetabular labral tears of the hip: examination and diagnostic challenges. J Orthop Sports Phys Ther. 2006;36(7):503-15. http:// dx.doi.org/10.2519/jospt.2006.2135

2. Crestani MV, Telöken MA, Gusmão PDF. Impacto femoroacetabular: uma das condições precursoras da osteoartrose do quadril. Rev Bras Ortop. 2006;41(8):285-93.

3. Martin RL, Irrgang JJ, Sekiya JK. The diagnostic accuracy of a clinical examination in determining intra-articular hip pain potential hip arthroscopy candidates. Arthroscopy. 2008;24(9):1013-8. http:// dx.doi.org/10.1016/j.arthro.2008.04.075

4. Banarjee P, McLean CR. Femoroacetabular impingement: a review of diagnosis and management. Curr Rev Musculoskelet Med. 2011;4(1):23-32. http://dx.doi.org/10.1007/s12178-011-9073-z

5. Ng VY, Arora N, Best TM, Pan X, Ellis TJ. Efficacy of surgery for femoroacetabular impingement: a systematic review. Am J Sports Med. 2010;38(11):2337-45. http://dx.doi.org/10.1177/036354651 0365530
6. Wahoff M, Ryan M. Rehabilitation after femoroacetabular impingement arthroscopy. Clin Sports Med. 2011;30:463-82. http://dx.doi.org/10.1016/j.csm.2011.01.001

7. Enseki KR, Draovitch P. Rehabilitation for hip arthroscopy. Oper Tech Orthop. 2010;20:278-81. http://dx.doi.org/10.1053/j. oto.2010.09.012

8. Spencer-Gardner L, Eischen JJ, Levy BA, Sierra RJ, Engasser WM, Krych AJ. A comprehensive five-phase rehabilitation programme after hip arthroscopy for femoroacetabular impingement. Knee Surg Sports Traumatol Arthrosc. 2014;22(4):848-59. http://dx.doi. org/10.1007/s00167-013-2664-Z

9. Stalzer S, Wahoff M, Scanlan M, Draovitch P. Rehabilitation after hip arthroscopy. Oper Tech Orthop. 2005;15:280-9. http://dx.doi. org/10.1053/j.oto.2005.06.007

10. Stalzer S, Wahoff M, Scanlan. Rehabilitation following hip arthroscopy. Clin Sports Med. 2006;25:337-57. http://dx.doi. org/10.1016/j.csm.2005.12.008

11. Enseki KR, Martin RL, Draovitch P, Kelly BT, Philippon MJ, Schenker ML. The hip joint: arthroscopic procedures and postoperative rehabilitation. J Orhopaed Sports Phys Ther. 2006;36(7):516-25. http://dx.doi.org/10.2519/jospt.2006.2138

12. Enseki KR, Martin R, Kelly BT. Rehabilitation after arthroscopic decompression for femoroacetabular impingement. Clin Sports Med. 2010;29:247-55. http://dx.doi.org/10.1016/j.csm.2009.12.007

13. Bennell KL, O'Donnell JM, Takla A, Spiers LN, Hunter DJ, Staples M, Hinman RS. Efficacy of a physiotherapy rehabilitation program for individuals undergoing arthroscopic management of femoroacetabular impingement - the FAIR trial: a randomised controlled trial protocol. BMC Musculoskelet Disord. 2014;26: 15-58. http://dx.doi.org/10.1186/1471-2474-15-58

14. Edelstein J, Ranawat A, Enseki KR, Yun RJ, Draovitch P. Post-operative guidelines following hip arthroscopy. Curr Rev Musculoskelet Med. 2012;5(1):15-23. http://dx.doi.org/10.1007/s12178-011-9107-6

15. Cheatham SW, Kolber MJ. Rehabilitation after hip arthroscopy and labral repair in a high school football athlete. Int J Sports Phys Ther. 2012;7(2):173-84

16. Beck M, Kalhor M, Leunig M, Ganz R. Hip morphology influences the pattern of damage to the acetabular cartilage: femoroacetabular impingement as a cause of early osteoarthritis of the hip. J Bone Joint Surg Br. 2005;87(7):1012-8. http://dx.doi.org/10.1302/0301620X.87B7.15203

17. Loudon JK, Reiman MP. Conservative management of femoroacetabular impingement (FAI) in the long distance runner. Phys Ther Sport. 2014;15(2):82-90. http://dx.doi.org/10.1016/j. ptsp.2014.02.004

18. Matheney T, Sandell L, Foucher K, Lamontagne M, Grodzinsky AJ, Peters CL. Motion analysis, cartilage mechanics, and biology in femoroacetabular impingement: current understanding and areas of future research. J Am Acad Orthop Surg. 2013;21 Suppl 1: S27-32. http://dx.doi.org/10.5435/JAAOS-21-07-S27

19. Sankar WN, Matheney TH, Zaltz I. Femoroacetabular impingement: current concepts and controversies. Orthop Clin North Am. 2013;44(4):575-89. http://dx.doi.org/10.1016/j.ocl.2013.07.003

20. Ganz R, Parvizi J, Beck M, Leunig M, Nötzli H, Siebenrock KA. Femoroacetabular impingement: a cause for osteoarthritis of the hip. Clin Orthop Relat Res. 2003;(417):112-20. 
21. Bardakos NV, Villar RN. Predictors of progression of osteoarthritis in femoroacetabular impingement: a radiological study with a minimum of ten years follow-up. J Bone Joint Surg Br. 2009;91(2):162-9. http://dx.doi.org/10.1302/0301-620X.91B2.21137

22. Van Houcke J, Pattyn C, Vanden Bossche L, Redant C, Maes JW, AudenaertEA. The pelvifemoral rhythm in cam-type femoroacetabular impingement. Clin Biomech (Bristol, Avon). 2014;29(1):63-7. http:// dx.doi.org/10.1016/j.clinbiomech.2013.10.019

23. Samora JB, Ng VY, Ellis TJ. Femoroacetabular impingement: a common cause of hip pain in young adults. Clin J Sport Med. 2011;21(1): 51-6. http://dx.doi.org/10.1097/JSM.0b013e318205dfde

24. Reid GD, Reid CG, Widmer N, Munk PL. Femoroacetabular impingement syndrome: an underrecognized cause of hip pain and premature osteoarthritis? J Rheumatol. 2010 Jul;37(7):1395-404. http://dx.doi.org/10.3899/jrheum.091186

25. Clohisy JC, Knaus ER, Hunt DM, Lesher JM, Harris-Hayes M, Prather $\mathrm{H}$. Clinical presentation of patients with symptomatic anterior hip impingement. Clin Orthop Relat Res. 2009;467(3):638-44. http:// dx.doi.org/10.1007/s11999-008-0680-y

26. Brisson N, Lamontagne M, Kennedy MJ, Beaulé PE. The effects of CAM femoroacetabular impingement corrective surgery on lower extremity gait biomechanics. Gait Posture. 2013;37(2):258-63. http://dx.doi.org/10.1016/j.gaitpost.2012.07.016

27. Casartelli NC, Maffiuletti NA, Item-Glatthorn JF, Staehli S, Bizzini M, Impellizzeri FM, Leunig M. Hip muscle weakness in patients with symptomatic femoroacetabular impingement. Osteoarthritis Cartilage. 2011;19(7):816-21. http://dx.doi.org/10.1016/j.joca. 2011.04.001

28. Rylander JH, Shu B, Andriacchi TP, Safran MR. Preoperative and postoperative sagittal plane hip kinematics in patients with femoroacetabular impingement during level walking. Am J Sports Med. 2011;39 Suppl:36S-42S. http://dx.doi.org/10.1177/ 0363546511413993

29. Kennedy MJ, Lamontagne M, Beaulé PE. The effect of CAM femoroacetabular impingement on hip maximal dynamic range of motion. J Orthop. 2009;1(1):41-50.

30. Kennedy MJ, Lamontagne M, Beaulé PE. Femoroacetabular impingement alters hip and pelvic biomechanics during gait: walking biomechanics of FAI. Gait Posture. 2009;30(1):41-4. http://dx.doi. org/10.1016/j.gaitpost.2009.02.008

31. Kennedy MJ, Lamontagne M, Beaulé PE. The effect of CAM FAI on hip and pelvic motion during maximum squat. Clin Orthop Relat Res. 2009;467(3):645-50. http://dx.doi.org/10.1007/s11999-008-0620-x

32. Philippon M, Schenker M, Briggs K, Kuppersmith D. Femoroacetabular impingement in 45 professional athletes: associated pathologies and return to sport following arthroscopic decompression. Knee Surg Sports Traumatol Arthrosc. 2007 Jul;15(7):908-14. http://dx.doi. org/10.1007/s00167-007-0332-x

33. Stulberg SD, Cordell LD, Harris WH, Ramsey PL, MacEwan GD. Unrecognized childhood hip disease: a major cause of idiopathic osteoarthritis of the hip. In: The Hip. Proceedings of the Third Open Scientific Meeting of The Hip Society, 1975. St. Louis, MO: C.V. Mosby; 1975.

34. Bedi A, Chen N, Robertson W, Kelly BT. The management of labral tears and femoroacetabular impingement of the hip in the young, active patient. Arthroscopy. 2008;24(10):1135-45. http://dx.doi. org/10.1016/j.arthro.2008.06.001
35. Chow RT, Johnson MI, Lopes-Martins RAB, Bjordal JM. Efficacy of low-level laser therapy in the management of neck pain: a systematic review and meta-analysis of randomised placebo or active treatment controlled trials. Lancet. 2009;374:1897-908. http://dx.doi.org/10.1016/S0140-6736(09)61522-1

36. Martin SS, Spindler KP, Tarter JW, Detwiler KB, Petersen HA. Cryotherapy: an effective modality for decreasing intraarticular temperature after knee arthroscopy. Am J Sports Med. 2001; 29(3):288-91.

37. Aimbire F, Albertini R, Pacheco MT, Castro-Faria-Neto HC, Leonardo PS, Iversen VV, Lopes-Martins RA, Bjordal JM. Low-level laser therapy induces dose-dependent reduction of TNFalpha levels in acute inflammation. Photomed Laser Surg. 2006;24(1):33-7. http:// dx.doi.org/10.1089/pho.2006.24.33

38. Bjordal JM, Couppé C, Chow RT, Tunér J, Ljunggren AE. A systematic review of low level laser therapy with location-specific doses for pain from joint disorders. Aust J Physiother. 2003;49(2):107-16. http:// dx.doi.org/10.1016/S0004-9514(14)60127-6

39. Konrath GA, Lock T, Goitz HT, Scheidler J. The use of cold therapy after anterior cruciate ligament reconstruction. A prospective, randomized study and literature review. Am J Sports Med. 1996;24(5):629-33. http://dx.doi.org/10.1177/036354659602400511

40. Raynor MC, Pietrobon R, Guller U, Higgins LD. Cryotherapy after ACL reconstruction: a meta-analysis. J Knee Surg. 2005;18(2): 123-9. http://dx.doi.org/10.1055/s-0030-1248169

41. Ni SH, Jiang WT, Guo L, Jin YH, Jiang TL, Zhao Y, Zhao J. Cryotherapy on postoperative rehabilitation of joint arthroplasty. Knee Surg Sports Traumatol Arthrosc. 2015 Nov;23(11):3354-61. http://dx.doi. org/10.1007/s00167-014-3135-X

42. Nadler SF, Weingand K, Kruse RJ. The physiologic basis and clinical applications of cryotherapy and thermotherapy for the pain practitioner. Pain Physician. 2004 Jul;7(3):395-9.

43. Knobloch K, Grasemann R, Spies M, Vogt PM. Midportion Achilles tendon microcirculation after intermittent combined cryotherapy and compression compared with cryotherapy alone: a randomized trial. Am J Sports Med. 2008;36(11):2128-38. http://dx.doi. org/10.1177/0363546508319313

44. Yanagisawa O, Homma T, Okuwaki T, Shimao D, Takahashi H. Effects of cooling on human skin and skeletal muscle. Eur J Appl Physiol. 2007;100(6):737-45. http://dx.doi.org/10.1007/s00421-007-0470-3

45. Karunakara RG, Lephart SM, Pincivero DM. Changes in forearm blood flow during single and intermittent cold application. J Orthop Sports Phys Ther. 1999;29(3):177-80. http://dx.doi.org/10.2519/ jospt.1999.29.3.177

46. Banerjee S, Kapadia BH, Issa K, McElroy MJ, Khanuja HS, Harwin SF, Mont MA. Postoperative blood loss prevention in total knee arthroplasty. J Knee Surg. 2013;26(6):395-400. http://dx.doi. org/10.1055/s-0033-1357491

47. Schaser KD, Disch AC, Stover JF, Lauffer A, Bail HJ, Mittlmeier T. Prolonged superficial local cryotherapy attenuates microcirculatory impairment, regional inflammation, and muscle necrosis after closed soft tissue injury in rats. Am J Sports Med. 2007;35(1):93-102. http://dx.doi.org/10.1177/0363546506294569

48. Glenn RE, Spindler KP, Warren TA, McCarty EC, Secic M. Cryotherapy decreases intraarticular temperature after $\mathrm{ACL}$ reconstruction. Clin Orthop Rel Res. 2004;421(9):268-72. http://dx.doi.org/10.1097/01. blo.0000126302.41711.eb 
49. Brosseau L, Milne S, Wells G, Tugwell P, Robinson V, Casimiro L, Pelland L, Noel MJ, Davis J, Drouin H. Efficacy of continuous passive motion following total knee arthroplasty: a metaanalysis. J Rheumatol. 2004;31(11):2251-64.

50. O'Driscoll SW, Giori NJ. Continuous passive motion (CPM): theory and principles of clinical application. J Rehabil Res Dev. 2000 Mar-Apr;37(2):179-88.

51. Salter RB. The physiologicic basis of continuous passive motion for articular cartilage healing and regeneration. Hand Clin. 1994; 10(2):211-9

52. Wilk M, Frańczuk B. Evaluating changes in the range of movement in the hip joint in patients with degenerative changes, before and after total hip replacement. Orthop Traumatol Rehabil. 2004; 30;6(3):342-9.

53. Dy CJ, Thompson MT, Crawford MJ, Alexander JW, McCarthy JC, Noble PC. Tensile strain in the anterior part of the acetabular labrum during provocative maneuvering of the normal hip. J Bone Joint Surg Am. 2008 Jul;90(7):1464-72. http://dx.doi.org/10.2106/ JBJS.G.00467

54. Safran MR, Giordano G, Lindsey DP, Gold GE, Rosenberg J, Zaffagnini S, Giori NJ. Strains across the acetabular labrum during hip motion: a cadaveric model. Am J Sports Med. 2011 Jul;39 Suppl:92S-102S. http://dx.doi.org/10.1177/0363546511414017

55. Philippon MJ1, Decker MJ, Giphart JE, Torry MR, Wahoff MS, LaPrade RF. Rehabilitation exercise progression for the gluteus medius muscle with consideration for iliopsoas tendinitis: an in vivo electromyography study. Am J Sports Med. 2011 Aug;39(8):177785. http://dx.doi.org/10.1177/0363546511406848

56. Freeman S, Mascia A, McGill S. Arthrogenic neuromusculature inhibition: a foundational investigation of existence in the hip joint. Clin Biomech. 2013;28(2):171-7. http://dx.doi.org/10.1016/j. clinbiomech.2012.11.014

57. Palmieri-Smith RM, Kreinbrink J, Ashton-Miller JA, Wojtys EM. Quadriceps inhibition induced by an experimental knee joint effusion affects knee joint mechanics during a single-legged drop landing. Am J Sports Med. 2007;35(8):1269-75. http://dx.doi. org/10.1177/0363546506296417

58. Selkowitz DM, Beneck GJ, Powers CM. Which exercises target the gluteal muscles while minimizing activation of the tensor fascia lata? Electromyographic assessment using fine-wire electrodes. J Orthop Sports Phys Ther. 2013 Feb;43(2):54-64. http://dx.doi.org/10.2519/ jospt.2013.4116

59. Richardson C, Hodges P, Hides J. Therapeutic exercise for lumbopelvic stabilization. London: Churchill Livingstone; 2004.

60. Akuthota V, Nadler SF. Core strengthening. Arch Phys Med Rehabil. 2004 Mar;85(3 Suppl 1):S86-92. http://dx.doi.org/10.1053/j. apmr.2003.12.005

61. Yazbek PM, Ovanessian V, Martin RL, Fukuda TY. Nonsurgical treatment of acetabular labrum tears: a case series. J Orthop Sports Phys Ther. 2011;41(5):346-53. http://dx.doi.org/10.2519/ jospt.2011.3225
62. Pennock AT, Philippon MJ, Briggs KK. Acetabular labral preservation: surgical techniques, indications, and early outcomes. Oper Tech Orthop. 2010; 20: 217-22. http://dx.doi.org/10.1053/j. oto.2010.09.013

63. Jayasekera N, Aprato A, Villar RN. Are crutches required after hip arthroscopy? A case-control study. Hip Int. 2013;23(3):269-73. http://dx.doi.org/10.5301/hipint.5000029

64. Behrens BJ, Michlovitz SL. Physical agents: theory and practice. Philadelphia: F.A Davis Company; 2006.

65. Kelly BT, Bedi A, Robertson CM, Dela Torre K, Giveans MR, Larson CM. Alterations in internal rotation and alpha angles are associated with arthroscopic CAM decompression in the hip. Am J Sports Med. 2012;40(5):1107-12. http://dx.doi.org/10.1177/ 0363546512437731

66. Kubiak-Langer M, Tannast M, Murphy SB, Siebenrock KA, Langlotz F. Range of motion in anterior femoroacetabular impingement. Clin Orthop Relat Res. 2007;458:117-24. http://dx.doi.org/10.1097/ blo.0b013e318031c595

67. Ellison JB, Rose SJ, Sahrmann SA. Patterns of hip rotation range of motion: a comparison between healthy subjects and patients with low back pain. Phys Ther. 1990;70(9):537-41.

68. Levine D, Whittle MW. The effects of pelvic movement on lumbar lordosis in the standing position. J Orthop Sports Phys Ther. 1996;24(3):130-5. http://dx.doi.org/10.2519/jospt.1996.24.3.130

69. Watanabe W, Sato K, Itoi E, Yang K, Watanabe H. Posterior pelvic tilt in patients with decreased lumbar lordosis decreases acetabular femoral head covering. Orthopedics. 2002;25(3):321-4.

70. Van Dillen LR, Bloom NJ, Gombatto SP, Susco TM. Hip rotation range of motion in people with and without low back pain who participate in rotation-related sports. Phys Ther Sport. 2008;9(2): 72-81. http://dx.doi.org/10.1016/j.ptsp.2008.01.002

71. Almeida GP, de Souza VL, Sano SS, Saccol MF, Cohen M. Comparison of hip rotation range of motion in judo athletes with and without history of low back pain. Man Ther. 2012;17(3):231-5. http://dx.doi. org/10.1016/j.math.2012.01.004

72. Le Beau RT, Nho SJ. The use of manual therapy post hip arthroscopy when an exercise based therapy approach has failed: a case report. J Orthop Sports Phys Ther. 2014;44(9):712-21. http://dx.doi. org/10.2519/jospt.2014.5047

73. Almeida TF, Roizenblatt S, Benedito-Silva AA, Tufik S. The effect of combined therapy (ultrasound and interferential current) on pain and sleep in fibromyalgia. Pain. 2003;104(3):665-72. http://dx.doi. org/10.1016/S0304-3959(03)00139-8

74. Renan-Ordine R1, Alburquerque-Sendín F, de Souza DP, Cleland JA, Fernández-de-Las-Pe-as C. Effectiveness of myofascial trigger point manual therapy combined with a self-stretching protocol for the management of plantar heel pain: a randomized controlled trial. J Orthop Sports Phys Ther. 2011;41(2):43-50. http://dx.doi. org/10.2519/jospt.2011.3504

75. Kolt GS, Snyder-Mackler L. Fisioterapia no esporte e no exercício. São Paulo: Revinter; 2008. 